J3eA, Journal sur l'enseignement des sciences et technologies de l'information et des systèmes, Volume 4, Hors-Série 2, 5 (2005)

DOI : http://dx.doi.org/10.1051/bib-j3ea:2005705

(C) EDP Sciences, 2005

Maquette de train alimenté par biberonnage : un outil d'enseignement et de recherche pluridisciplinaire

\author{
P. Barrade et A. Rufer
}

Laboratoire d'Électronique Industrielle

Institut des Sciences de l'Énergie

Faculté des Sciences et Techniques pour l'Ingénieur

École Polytechnique Fédérale de Lausanne

CH-1015 Lausanne, Suisse 


\title{
Maquette de train alimenté par biberonnage : un outil d'enseignement et de recherche pluridisciplinaire
}

\author{
P. Barrade, A. Rufer \\ Laboratoire d'Electronique Industrielle (LEI), \\ Institut des Sciences de l'Energie (ISE), Faculté des Sciences et Techniques pour l'Ingénieur \\ (STI), Ecole Polytechnique Fédérale de Lausanne, (EPFL) \\ CH-1015 Lausanne, Suisse \\ philippe.barrade@epfl.ch
}

\section{Résumé :}

Le cursus universitaire des étudiants de l'EPFL comprend, outre des cours magistraux, des travaux dirigés et des travaux pratiques, la réalisation de projets de semestre et de diplômes. Les étudiants ont ainsi l'occasion, au travers de ces projets, soit de parfaire leurs connaissances soit d'acquérir en auto-apprentissage de nouvelles compétences. De tels projets représentent ainsi des moments forts dans la formation des ingénieurs, d'autant qu'ils sont effectués en laboratoires de recherche, et que les thèmes proposés sortent du caractère purement académique pour embrasser une problématique de recherche ou de développement propre à susciter la motivation des étudiants. Le propos de cette contribution est de présenter une maquette de train, alimenté par supercondensateurs. Il s'agit en premier lieu d'un thème de recherche du LEI. Mais la réalisation de cette maquette a été confiée à des étudiants, qui se sont succédés et qui ont dû mettre en œuvre des compétences pluridisciplinaires pour parvenir au résultat présenté ici.

Mots clés : Recherche, Energie, Stockage, Enseignement, Auto-apprentissage, Projets, Pluridisciplinaire

\section{Introduction}

L'Ecole Polytechnique Fédérale de Lausanne (EPFL) est une institution Suisse, dont la double vocation est d'une part la recherche, et d'autre part l'enseignement.

Coté recherche, l'EPFL est divisé en cinq facultés, au sein desquelles sont développés des programmes transdisciplinaires qui favorisent les recherches à l'interface des disciplines traditionnelles. De fait chaque Faculté est divisée en institut, chaque institut regroupant plusieurs laboratoires. Au sein de l'Institut des Sciences de L'Energie (ISE), le Laboratoire d'Electronique Industrielle (LEI) développe ses compétences dans les domaines de l'électronique industrielle et de l'électronique de puissance.

Les activités d'enseignements sont réparties de manière orthogonale entre les différentes facultés, qui doivent assurer la formation d'élèves ingénieurs se répartissant dans 13 filières de formation, dont la section électricité. L'essentiel des enseignements donnés pour la section électricité est assuré par les enseignants de la Faculté des Sciences et techniques pour l'Ingénieur (STI), à laquelle le LEI est rattaché. Les tâches d'enseignement affectées au LEI se répartissent en cours ex-cathedra, travaux dirigés et exercices dans les domaines de l'électronique de puissance, de l'électronique industrielle, et du stockage de l'énergie.

Le LEI doit en outre accueillir chaque semestre des étudiants dans le cadre de projets de diplôme et de semestre. Le but recherché par de tels projets est de permettre aux étudiants de parfaire ou d'acquérir des connaissances. Au travers d'une étude demandant à l'étudiant non seulement la mise en œuvre d'un savoir technique et scientifique, mais également l'apprentissage d'une méthode de travail lui permettant de mener à bien le projet, il s'agit pour l'étudiant de gagner notamment en autonomie, face à une problématique devant laquelle la réponse n'est pas aussi certaine que dans des séances d'exercice. Ce dernier point est d'autant plus intéressant que les thèmes d'études proposés dans ces projets ne sont pas des thèmes purement académiques, «pour faire réfléchir », mais des thèmes fortement couplés aux activités de recherche du laboratoire d'accueil.

L'objet de cette contribution est de décrire une des activités de recherche du LEI, fortement liée aux activités pédagogiques, ayant trait au stockage de l'énergie électrique. Il sera décrit dans un premier temps le concept étudié, se référant à l'alimentation séquentielle par biberonnage de véhicules électriques. Dans un deuxième temps, il sera présenté le lien entre cette activité recherche et la formation des élèves ingénieurs de l'EPFL, par la réalisation de démonstrateurs dans le cadre de projets de semestre et de diplômes. Outre les objectifs pédagogiques de tels projets, un élément essentiel est de présenter aux étudiants des sujets attrayants les poussant à s'intéresser au génie électrique en général, et à la problématique majeure de la gestion de l'énergie en particulier.

\section{Alimentation de véhicules par biberonnage} 2.1. Stockage de l'énergie 
De récents développements ont conduit à l'apparition de nouveaux composants dédiés au stockage de l'énergie électrique: les supercondensateurs.

Ces composants sont caractérisés par des densités en énergie nettement supérieures à celles des condensateurs classiques (on parle de capacités pouvant atteindre les $3600 \mathrm{~F}$ ), et des densités en puissance accrues par rapport aux batteries.

Ainsi, même si la densité en énergie des supercondensateurs reste inférieure à celle des batteries dans un rapport 10, ces nouveaux composants offrent de nouvelles perspectives dans toute application où le stockage d'énergie n'impose pas de contraintes majeure vis-à-vis de la densité en énergie, mais où la disponibilité en puissance

\subsection{Alimentation d'un véhicule électrique}

Une étude est actuellement en cours au LEI, où l'on envisage le recours à la technologie des supercondensateurs pour rendre autonome des bus électriques.

L'origine de cette problématique est issue de considérations relatives à l'alimentation de bus électriques par caténaires, qu'il serait judicieux de supprimer dans les centres hyper-urbain ou en site classé. Cette problématique est schématisée sur la fig. 1 , où l'alimentation par caténaire est maintenue sur une portion péri-urbaine du parcourt, et supprimée sur une portion correspondant à un centre ville.

La densité en énergie des supercondensateurs n'est à ce jour pas assez performante pour garantir

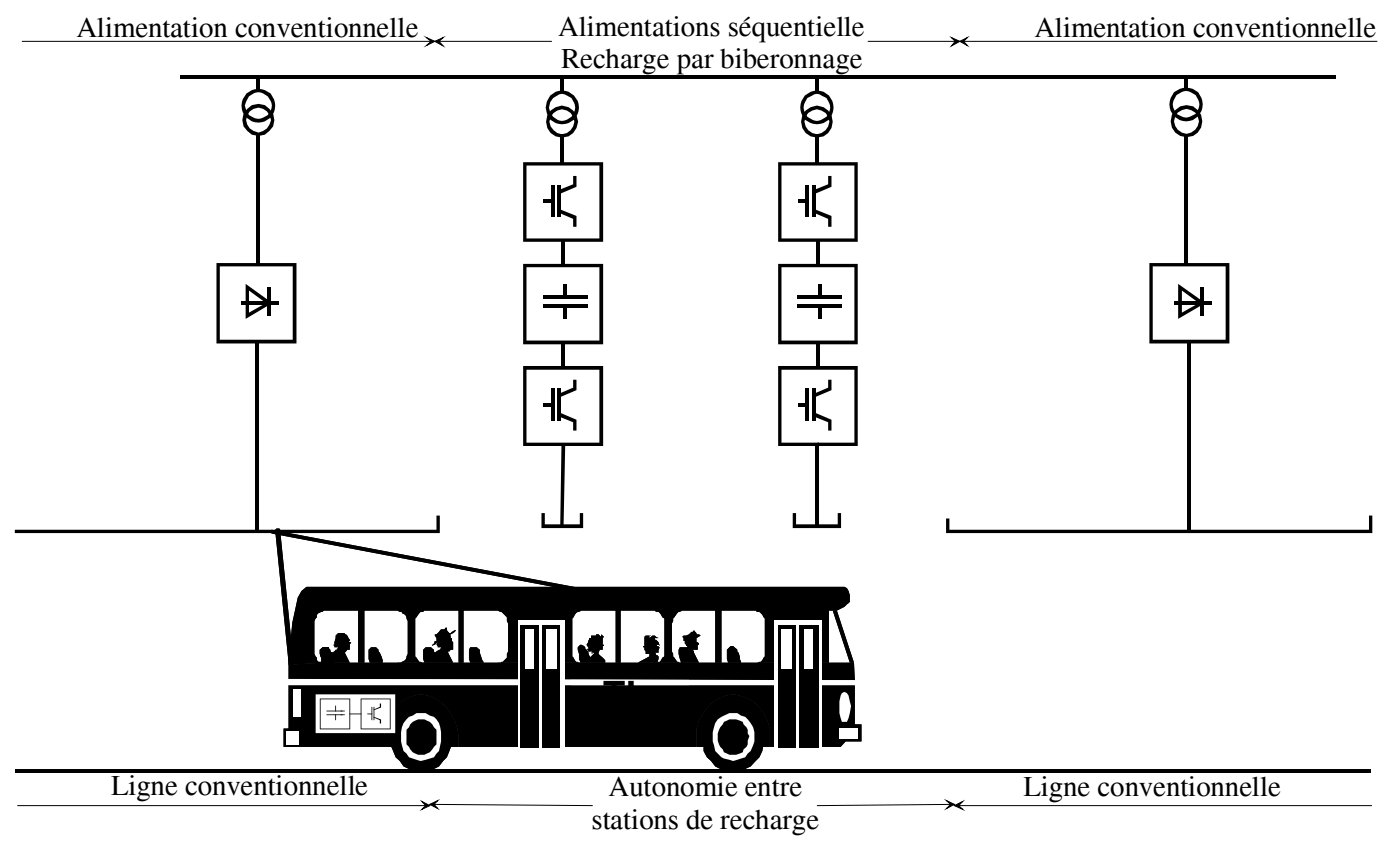

Fig. 1: Autonomie réduite d'un bus électrique

requise n'est pas compatible avec la technologie des batteries.

Dernier avantage des supercondensateurs, le nombre de cycles charge/décharge élevé (100'000) par rapport à ceux caractérisant les batteries (1000 2500).

Plusieurs applications font aujourd'hui l'objet d'études sérieuses, pour lesquelles on envisage l'utilisation des supercondensateurs en tant que source d'énergie principale pour un système (alimentations sans interruption, outils autonomes), ou en tant que réservoir tampon en énergie pour limiter les contraintes en puissance sur une source d'énergie: association supercondensateurs et batteries ou pile à combustible, maintien de la tension sur des réseaux de distribution non rigides, diminution des contraintes en puissance imposées sur un réseau par un ascenseur, etc...[1] l'autonomie du bus sur la totalité du parcourt en mode autonome. En revanche, le bus doit s'arrêter périodiquement aux stations pour embarquer/débarquer les usagers. Les temps d'arrêts sont largement compatibles avec le temps nécessaire à la recharge des supercondensateurs embarqués à bord du véhicule (une dizaine de secondes), ce qui permet de dimensionner le banc de supercondensateurs pour n'assurer l'autonomie du véhicule qu'entre deux stations d'arrêt.

Des études réalisées à Lausanne $(\mathrm{CH})$ montrent qu'un banc de supercondensateurs de $1.6 \mathrm{~m}^{3}$ peut suffire à cela. Des résultats de simulation, basés sur des mesures effectuées sur un véhicule sont proposés sur Fig. 2.

La courbe bleue est la puissance demandée par un véhicule électrique. La courbe verte est la puissance fournie par le banc de supercondensateurs 


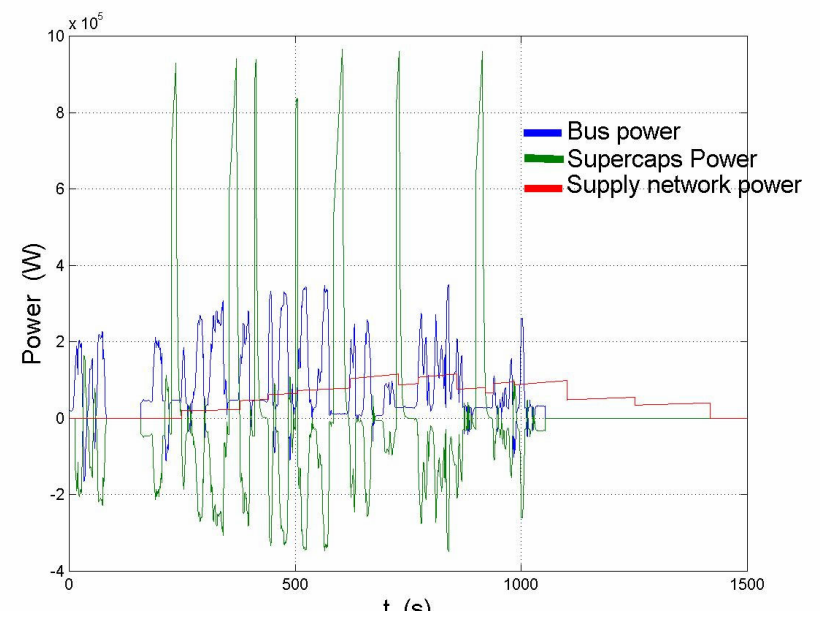

Fig. 2: Alimentation d'un bus par supercondensateurs

embarqués à bord du véhicule. Cette puissance est l'image de la puissance demandée par le bus, sauf lors des phases de recharge rapide des supercondensateurs embarqués, où le stockage de $17 \mathrm{MJ}$ en une dizaine de secondes nécessite des puissances de recharge de l'ordre du mégawatt. Ceci est incompatible avec la disponibilité en puissance des réseaux.

Pour palier à ces contraintes, il apparaît sur la Fig. 1 des supercondensateurs fixes dans chacune des stations de recharge. L'énergie y étant stockée au préalable, le transfert rapide sous puissance élevée de l'énergie entre le banc de supercondensateurs fixes et ceux embarqués se fait sans contrainte sur le réseau d'alimentation moyenne tension. Lorsque les supercondensateurs fixes sont rechargés et que le bus quitte la station, les supercondensateurs fixes doivent être rechargés pour préparer la venue du bus suivant. On dispose alors de 10 minutes pour réaliser cela, ce qui permet de ne prélever sur le réseau qu'une puissance de $20 \mathrm{~kW}$. De fait, le courbe rouge de Fig. 2 est la somme des puissances prélevées sur le réseau de chacune des sept stations de recharge : la puissance absorbée au réseau est la puissance moyenne demandée par le bus, et est nettement inférieure à la puissance maximale demandée par le véhicule en fin d'accélération.

On illustre ainsi l'aptitude des supercondensateurs à jouer le rôle de réservoirs tampon en énergie afin de limiter les contraintes en puissance sur une source d'énergie. Ce concept de recharge séquentielle de supercondensateurs embarqués par des bancs de supercondensateurs fixes est appelé «biberonnage » [2].

\section{Projets pédagogiques}

\subsection{Contexte}

Le projet énoncé ci-dessus est d'abord et avant tout un projet de recherche. Des études théoriques ont été développées. Au stage actuel de l'étude, des validations expérimentales devaient être réalisées afin de juger de la viabilité des concepts proposés.
Cependant, vu le nombre de supercondensateurs mis en œuvre, et leur prix encore élevé, une validation à l'échelle 1 était impossible.

Pour cette raison, nous nous sommes orientés vers la réalisation d'un prototype à échelle réduite, basé sur un modèle réduit de train de type LGB (Fig. 3).

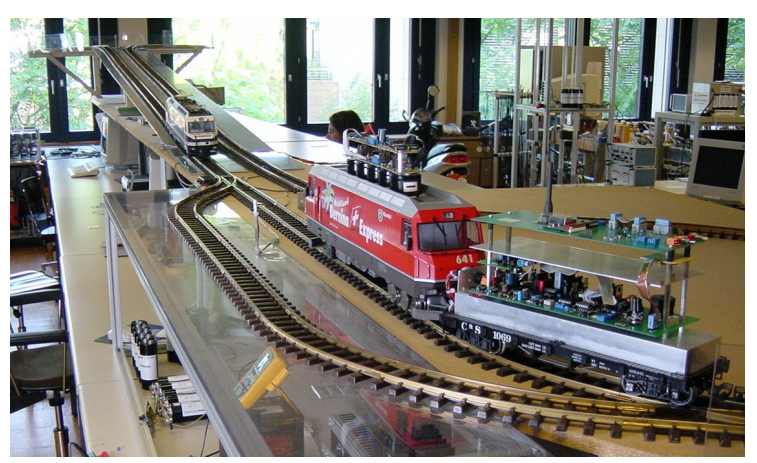

Fig. 3: Maquette à échelle réduite

Il est apparut ainsi intéressant de confier le développement de ce démonstrateur aux étudiants de l'EPFL. Ces derniers doivent en effet effectuer deux projets de semestre et un projet de diplôme dans leur cursus. Il semblait donc adéquat de leur proposer un sujet d'étude ludique, mais présentant un aspect novateur indéniable.

\subsection{Travaux réalisés}

Les projets de réalisation du démonstrateur présenté Fig. 3 ont consisté dans un premier temps à adapter la maquette, afin de rendre les entraînements du train réversibles, et mettre en évidence les propriétés du freinage du véhicule par récupération d'énergie. Un premier étudiant s'est donc chargé de re-concevoir les bogies du train. Le résultat est présenté Fig. 4.

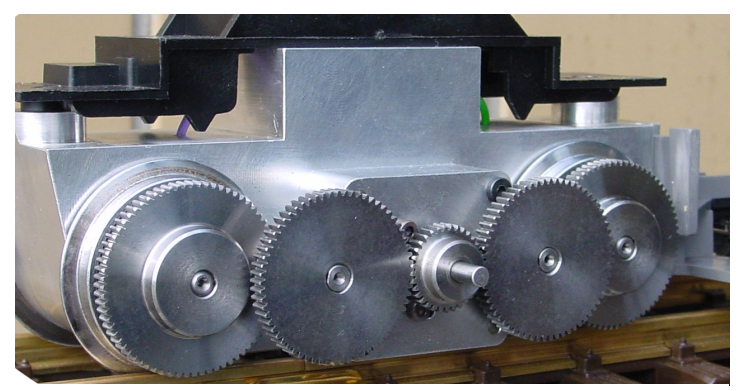

Fig. 4: Bogies du train

Un deuxième travail consistait à concevoir l'alimentation d'interface entre le banc de supercondensateurs embarqués et les moteurs du train. Ce travail a été suivi par deux autres projets : conception des cartes de communication radiofréquences permettant de piloter le train à distance à partir d'un ordinateur, et de centraliser toutes les données enregistrées lors d'un cycle de fonctionnement complet.

Ces études se sont poursuivies, et se poursuivent encore pour améliorer les protocoles de 
communication entre le train et l'ordinateur de contrôle, par le biais d'interfaces graphiques, ainsi que présenté Fig. 5.

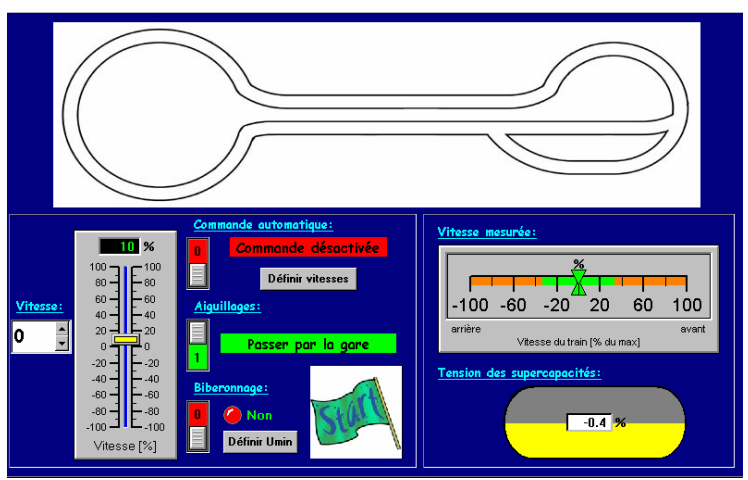

Fig. 5: Interface graphique

La station de recharge par biberonnage est actuellement en cours de réalisation, et fera l'objet d'un nouveau projet de semestre dans le courant de l'hiver 2003-2004.

$\mathrm{Au}$ résultat, nous disposons à présent d'un démonstrateur réaliste bien qu'en cours d'évolution, qui permet d'illustrer la problématique de tout véhicule électrique dans le cadre de travaux pratiques, et assez réalistes pour sensibiliser les étudiants au thème de la gestion d'énergie dans un système complexe, tout en permettant la validation expérimentale des concepts de recherche énoncés précédemment.

\subsection{Recherche et pédagogie}

Il est intéressant d'observer comment une telle étude combine des aspects relatifs à la fois à la recherche et à la pédagogie.

Un thème de recherche ambitieux permet avantageusement de définir un fil conducteur générant de thèmes de travaux pour les étudiants. Ces derniers ne travaillent donc pas sur un thème purement académique, mais sur une problématique propre visant à développer leur intérêt sur des disciplines particulières.

Il ne s'agit plus de développer «pour développer », mais de concevoir pour répondre à des cahiers des charges basés sur des considérations techniques avant que d'être basés sur des considérations uniquement pédagogiques. Les travaux effectués ne sont alors plus à buts applicatifs des notions vues en cours, ce qui permet de sortir d'un contexte quelque fois trop scolaire.

Il est de plus à noter que de tels thèmes d'étude, portant sur des systèmes complexes, nécessitent de la part des étudiants des considérations plus étendues que sur un projet moins ambitieux : développer un tel démonstrateur a nécessité des travaux dans les domaines de la mécanique, de l'électronique de puissance, de l'électronique industrielle, du traitement du signal, de la programmation, etc... Face à la pluridisciplinarité d'un tel projet, un effort d'encadrement doit être fourni afin que les étudiants soient guidés et tirent en enrichissement par rapport aux domaines où ils sont le moins compétent.

Enfin, il doit être précisé que pas moins de 5 étudiants et 4 apprentis se sont succédés sur ce projet jusqu'à présent. Un sixième étudiant prendra le relais au semestre d'hiver 2003-2004. Un effort important doit donc être consacré à la nécessité de bien documenter le travail effectué, afin qu'il puisse être repris facilement par l'étudiant suivant. Ceci rentre pleinement dans la formation d'un futur ingénieur, qui doit être capable de communiquer efficacement son travail.

Par ailleurs, les étudiants sont amenés à communiquer eux-mêmes les résultats obtenus dans des conférences [3].

\section{Conclusion}

L'apparition de nouveaux composants tels que les supercondensateurs permet d'envisager aujourd'hui de nouveaux concepts pour l'alimentation de véhicules électriques, tels que l'alimentation par biberonnage.

Par leur double vocation de recherche et d'enseignement, les laboratoires de l'EPFL sont en mesure de proposer aux étudiants des projets d'étude fortement liés à leurs activités de recherche.

L'étude menée par le LEI autour du concept de biberonnage a ainsi permis de proposer aux étudiants la réalisation d'un démonstrateur, leur donnant ainsi l'occasion d'approfondir leurs connaissances et d'en acquérir de nouvelles, dans un contexte très motivant pour eux. Ceci, dans le cadre d'une étude portant sur un système complexe, mettant en œuvre des notions d'ordre pluridisciplinaires.

\section{Bibliographie}

1. A. Rufer, P. Barrade, Stockage d'énergie électrique par super-condensateurs, solutions de l'électronique de puissance et applications, EF'2001 : Colloque Electrotechnique du Futur, 14-15 November, Palais des congrès, Nancy, France.

2. P. Barrade, A. Rufer, Power smoothing and high power fast energy exchange between storage systems, International Conference on Quality and ecurity of Electrical Supply, 26-27 February, The Oxford Belfry, Thame, ERA Technology LTD, Surrey KT22 7SA, UK.

3. P.-A. Giroud, D. Hotellier, Supercapacitive Storage - Better consumption of electrical energy, FIDIC Meeting 2001 : Fédération Internationale Des Ingénieurs Conseil, 2-5 September, Montreux, Switzerland. 